\title{
Difference in pain and accuracy of two hyaluronic acid injection techniques for symptomatic knee osteoarthritis in overweight patients
}

\author{
Roberto D'Alessandro ID, Paolo Falsetti ID , Edoardo Conticini ID , Suhel Gabriele Al Khayyat ID, \\ Marco Bardelli ${ }^{D}$, Caterina Baldi ${ }^{\text {ID }}$, Stefano Gentileschi ${ }^{1 D}$, Luca Cantarini ${ }^{\text {ID }}$, Bruno Frediani ${ }^{\text {ID }}$ \\ Department of Medicine, Surgery and Neurosciences, Rheumatology Unit, University of Siena, Italy
}

\begin{abstract}
Objectives: To evaluate differences of injection related pain, and the accuracy of the techniques in two groups of overweight patients, performing the anterolateral approach for one group (G1) and the superolateral approach for the second group (G2).

Material and methods: In the study, 126 knee joints from 86 osteoarthritis (OA) patients were evaluated. Inclusion criteria were body mass index $(\mathrm{BMI}) \geq 25$, absence of effusion and coagulopathy. Pain evaluation during injection was evaluated with Visual Analogue Scale (VAS), while accuracy of the procedure was evaluated with ultrasound (US).

Results: The patients' mean age was $69.9 \pm 9$.01, VAS for G1 group was $1.71 \pm 1.89$, for G2 group was $1.74 \pm 1.31$. Mean BMI was $29.69 \pm 2.86$, for $\mathrm{G} 1$ group was $28.29 \pm 3.29$, for $\mathrm{G} 2$ group was $30.32 \pm 2.41$. No adverse events (AE) occurred in both studied groups. The accuracy rate of the procedure was $69.1 \%$ for G1 (38/55 knees), $95.7 \%$ for G2 (68/71 knees). No significant difference was found in VAS pain score between $\mathrm{G} 1$ and $\mathrm{G} 2$ group $(p=0.45)$. We found the significant correlation between BMI and VAS pain score in anterolateral accesses (G1) $(r=0.51 ; p<0.005)$. No correlation was found between age and VAS pain score in anterolateral access (G1). For the superolateral access (G2), no correlation was found for age or BMI and VAS pain score.

Conclusions: Hyaluronic acid injection is safe therapeutic option for knee OA with no significant differences between anterolateral and superolateral approaches in terms of pain in overweight patients. However, higher BMI seems to be a predictor of pain in anterolateral access, and the superolateral approach should be preferred in this group of patients.
\end{abstract}

Key words: knee osteoarthritis, pain, overweight, injection, hyaluronic acid.

\section{Introduction}

Knee osteoarthritis (OA) is one of the most common chronic diseases, affecting $24 \%$ of the adult population [1]. Diagnosis of knee OA relies on clinical [2] and radiological findings [3].

Treatment of OA is firstly based on a non-pharmacological approach such as weight loss, as recommended by EULAR [4]. Overweight seems to be correlated with $\mathrm{OA}$ even for immunologic factors other than the more evident biomechanical implications. Indeed, obesity and adipocytokines produced by systemic visceral adipose tissue and locally by the infrapatellar bursa, also known as Hoffa's fat pad, might play an important role in the development and progression of knee OA [5].

Moreover, some adipocytokines (serum interleukin 6 and leptin) and synovial fluid leptin seem to be positively associated with body mass index (BMI) and preoperative pain severity in knee OA [6]. Second line treatment is based on topical and oral analgesic such as paracetamol, nonsteroidal anti-inflammatory drugs and symptomatic

Address for correspondence:

Roberto D’Alessandro, Department of Medical Sciences, Surgery and Neurosciences, Rheumatology Unit, University of Siena,

Azienda Ospedaliero-Universitaria Senese, Viale Bracci 16, 53100 Siena, Italy, e-mail: rt.dalessandro@gmail.com,

ORCID: https://orcid.org/0000-0002-8350-7581

Submitted: 27.04.2020; Accepted: 04.02.2021 
slow-acting drugs for osteoarthritis, followed by intraarticular (IA) viscosupplementation with hyaluronic acid (HA) [7].

For knee injections, both with corticosteroids and $\mathrm{HA}$, two techniques are usually performed by physicians: the superolateral and the anterolateral approach. The superolateral approach, with the leg in extension, seems to be the most widespread and accurate injection technique [8]. The anterolateral approach is performed with a $90^{\circ}$ flection of the knee, allowing the patient to remain in a sitting position, despite its lower accuracy [9].

In clinical practice, knee injection is a very well tolerated therapeutic option. In the literature, few papers have assessed injection related pain in knee OA, with contrasting outcomes between pain difference for the two techniques [10, 11].

The aim of this study was to evaluate differences of injection related pain using Visual Analogue Scale (VAS) [12], and the accuracy of the techniques in two groups of overweight patients, performing the anterolateral approach for one group and the superolateral approach for the second group.

\section{Material and methods}

We enrolled all overweight patients presenting to the Rheumatology Unit injection service of our hospital between September 2019 and March 2020. A total of one hundred twenty-six knees of 84 patients were divided in two groups, G1 (55 knees) and G2 (71 knees).

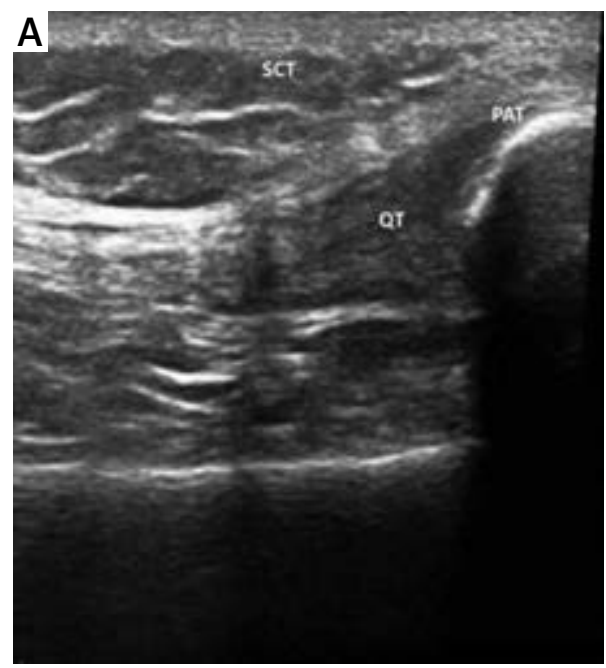

Inclusion criteria were knee osteoarthritis confirmed by clinical and radiological evidence (Kellgren-Lawrence score $\geq 2$ ) [3] and BMI $\geq 25$. Exclusion criteria were bleeding disorders, knee effusion, joint prothesis. Moderate molecular weight HA (range 1500-3000 kDa) was injected [13]. Pain during injection was evaluated using the VAS for pain (0-10), where 0 indicates no pain and 10 indicates the most severe pain. Before injection, meticulous aseptic technique was performed.

Procedure accuracy was evaluated by ultrasound (US). Ultrasonography was performed with an Esaote MyLab. Twice machine equipped with a multifrequency 6-18 MHz linear probe. Ultrasound examination permitted the direct visualization of viscous hyperechoic fluid, often associated with hyperechoic foci and air bubbles, spreading into the joint capsule of the suprapatellar recess [14] (Fig. 1).

The suprapatellar knee recess was scanned with an anterior longitudinal approach, proximal to the superior pole of the patella. The anterolateral approach was used for G1. The superolateral approach was used for G2. For all the injections, 21-gauge needles were used. The anterolateral access (G1) was performed with the patient in a sitting position with the knee flexed to $90^{\circ}$ at $1 \mathrm{~cm}$ proximal to the joint line, lateral to the patellar tendon, directing the needle towards the inter-condylar notch (Fig. 2 A). The superolateral access (G2) was performed with the patient in supine position, extending the knee; the needle was inserted $1 \mathrm{~cm}$ above and $1 \mathrm{~cm}$ lateral to the superolateral margin of the patella at a $90^{\circ}$ angle to the femoral condyle with a concomitant subluxation

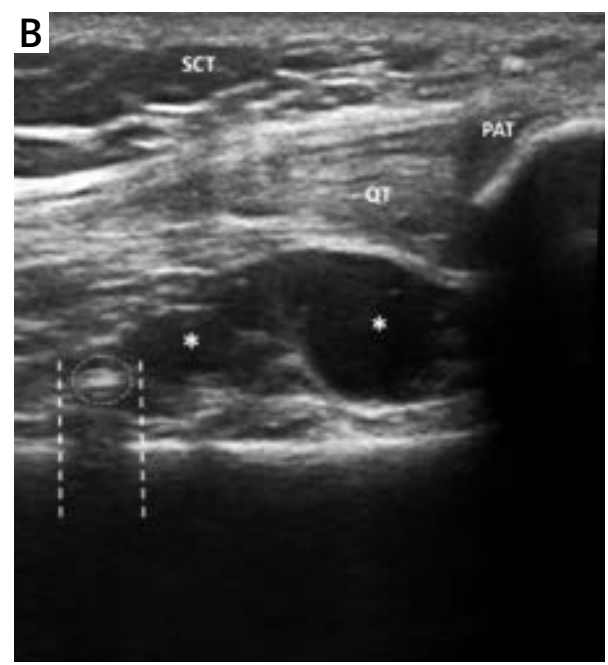

Fig. 1. Ultrasound examination performed before IA injection demonstrating absence of fluid in suprapatellar recess (A). Ultrasound examination performed after IA injection demonstrates a small amount of hypoechoic fluid $\left(^{*}\right)$ in the suprapatellar recess (B). Some hyperechoic foci (dotted circle) with mild acoustic posterior shadow (dashed line) are often visible after IA HA injection, because of the viscous nature of HA and the presence of bubbles.

SCT - subcutaneous tissue, QT - quadricipital tendon, PAT - patella. 


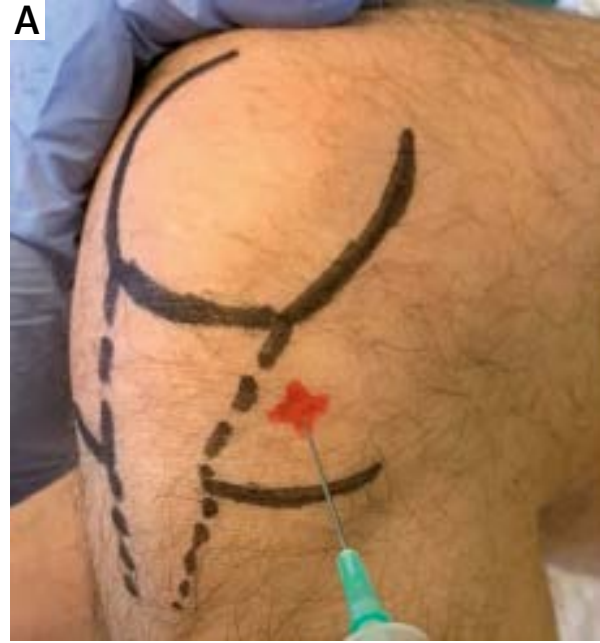

Fig. 2. Anterolateral access (A), superolateral access

of patella (Fig. 2 B). Both techniques were executed with no US aid and performed by the same trained physician.

\section{Statistical analysis}

The Mann-Whitney test for unpaired data was used to compare the VAS pain score between G1 and G2. Spearman's test was performed to assess the correlation between VAS pain score and BMI, age, gender, and site of injection. Linear regression was performed for each access to evaluate the relationship between VAS pain score and the independent variables.

\section{Ethical approval}

The study was approved by the local Ethical Committee (number: 15363 September 16th 2019) and carried out in accordance with the Helsinki Declaration, revised in 2013.

All patients gave their informed consent.

\section{Results}

A total of 126 knees of 84 patients were evaluated. Twenty-six patients were male, 58 female. Mean age was $69.9 \pm 9$.01. Mean VAS pain score for the anterolateral approach (G1) was $1.71 \pm 1.89$. Mean VAS pain score for the superolateral approach (G2) was $1.74 \pm 1.31$. Mean BMI was $29.69 \pm 2.86$. Mean BMI of G1 was $28.29 \pm 3.29$. Mean BMI of $\mathrm{G} 2$ was $30.32 \pm 2.41$.

No adverse events (AE) occurred in either group. The accuracy rate of the procedure, evaluated by US after the injection, was $69.1 \%$ for G1 (38/55 knees), $95.7 \%$ for G2 (68/71 knees). No significant difference was found in VAS pain score between G1 and G2 ( $p=0.45)$. We found a significant correlation between BMI and VAS pain score in anterolateral accesses (G1) $(r=0.51$ $p<0.005)$. No correlation was evidenced between age

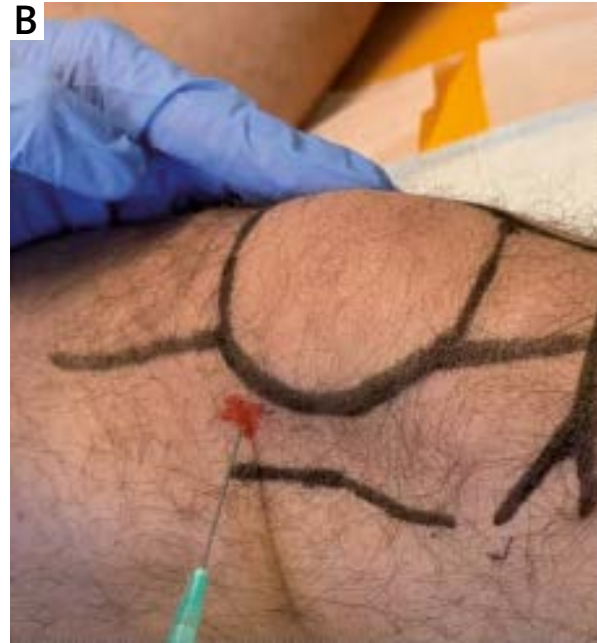

(B). Entrance site is marked in the picture.

Table I. Demographic and clinical characteristics of patients

\begin{tabular}{lc}
\hline Total patients $(n)$ & 84 \\
\hline Male/Female $(n)$ & $69.9 \pm 9.01$ \\
\hline Mean age \pm DS & 126 \\
\hline Total knees & $55 / 71$ \\
\hline G1/G2 $(n)$ & $1.71 \pm 1.89 / 1.74 \pm 1.31$ \\
\hline VAS G1/VAS G2 \pm DS & $29.69 \pm 2.86$ \\
\hline BMI total \pm DS & $28.29 \pm 3.29 / 30.32 \pm 2.41$ \\
\hline BMI G1/G2 \pm DS & 0.45 \\
\hline$p$-value VAS G1 vs. G2 & $<0.005$ \\
\hline$p$-value VAS-BMI & \\
(linear regression) G1 & None \\
\hline Adverse event G1 and G2 & $69.1 / 95.7$ \\
\hline Accuracy G1/G2 $(\%)$ &
\end{tabular}

and VAS pain score in anterolateral access (G1). For the superolateral access (G2), no correlation was found for age or BMI and VAS pain.

Demographic and clinical characteristics of our cohort are reported in Table I.

\section{Discussion}

We found no difference in injection related pain between anterolateral and superolateral access of knee injection of $\mathrm{HA}$ in overweight patients. An increase in $\mathrm{BMI}$ seems to be a predictor of pain in anterolateral access. Our pain VAS results are partially in contrast with previous findings: Chavez-Chiang et al. [10] reported a mean VAS pain score of 4.8 for anterolateral access, while Lee et al. [11] found that the anterolateral approach is less painful than superolateral. We could hypothesize that such differences might be related to 
the use of a thinner 21-gauge needle than Lee's cohort [11]; moreover, operator expertise and grade of local joint inflammation may be taken into account in order to explain the different outcomes.

We assessed the relation between BMI and pain in anterolateral access. This evidence could be explained locally, with a major production of adipocytokines [5] partially due to the plentiful of subcutaneous tissue in overweight patients rather than Hoffa's fat pad, whose volume seems not related to weight [15]. The influence of meniscal lesions should be considered too, since adipocytokines may have an influence on its pathogenesis [16].

We found an accuracy rate, evidenced by US, of $95.7 \%$ for superolateral access, while a lower rate of $69.1 \%$ was evidenced for anterolateral access, as recently reported [8]. This finding could be explained by the different and complex anatomical structure between the anterior and lateral compartment of the knee, which might even present differences between both knees of the same patients [17].

Finally, in relationship with the different knee compartments it is possible to speculate that HA may not have a homogeneous distribution in the whole joint [18]. In our cohort, no patients reported AEs, confirming the safety of HA injection [19].

\section{Conclusions}

Hyaluronic acid injection is a widespread and safe therapeutic option for knee OA with no significant differences between anterolateral and superolateral approaches in terms of pain in overweight patients. However, higher BMI seems to be a predictor of pain in anterolateral access, and the superolateral approach should be preferred for its accuracy.

\section{The authors declare no conflict of interest.}

\section{References}

1. Pereira D, Peleteiro B, Araujo J, et al. The effect of osteoarthritis definition on prevalence and incidence estimates: a systematic review. Osteoarthritis Cartilage 2011; 19: 1270-1285, DOI: 10.1016/j.joca.2011.08.009.

2. Nazari G. Knee osteoarthritis. J Physiother 2017; 63: 188, DOI: 10.1016/j.jphys.2017.04.004.

3. Kellgren JH, Lawrence JS. Radiological assessment of osteoarthrosis. Ann Rheum Dis 1957; 16: 494-502, DOI: 10.1136/ ard.16.4.494.

4. Fernandes L, Hagen KB, Bijlsma JWJ, et al. EULAR recommendations for the non-pharmacological core management of hip and knee osteoarthritis. Ann Rheum Dis 2013; 72: 1125-1135, DOI: 10.1136/annrheumdis-2012-202745.

5. Belluzzi E, El Hadi H, Granzotto M, et al. Systemic and local adipose tissue in knee osteoarthritis. J Cell Physiol 2017; 232: 1971-1978, DOI: 10.1002/jcp.25716.
6. Azim S, Nicholson J, Rebecchi MJ, et al. Interleukin-6 and leptin levels are associated with preoperative pain severity in patients with osteoarthritis but not with acute pain after total knee arthroplasty. Knee 2018; 25: 25-33, DOI: 10.1016/ j.knee.2017.12.001.

7. Bruyère $O$, Cooper $C$, Pelletier JP, et al. A consensus statement on the European Society for Clinical and Economic Aspects of Osteoporosis and Osteoarthritis (ESCEO) algorithm for the management of knee osteoarthritis-From evidence-based medicine to the real-life setting. Semin Arthritis Rheum 2016; 45 (4 Suppl): S3-S11, DOI: 10.1016/j.semarthrit.2015.11.010.

8. Hermans J, Bierma-Zeinstra SM, Bos PK, et al. The most accurate approach for intra-articular needle placement in the knee joint: a systematic review. Semin Arthritis Rheum 2011; 41: 106-115, DOI: 10.1016/j.semarthrit.2011.02.007.

9. Jackson DW, Evans NA, Thomas BM. Accuracy of needle placement into the intra-articular space of the knee. J Bone Joint Surg Am 2002; 84: 1522-1527, DOI: 10.2106/00004623200209000-00003.

10. Chavez-Chiang CE, Sibbitt WL Jr, Band PA, et al. The highly accurate anteriolateral portal for injecting the knee. Sports Med Arthrosc Rehabil Ther Technol 2011; 3: 6, DOI: 10.1186/17582555-3-6.

11. Lee SY, Gn KK, Chung BJ, et al. Anterolateral portal is less painful than superolateral portal in knee intra-articular injection. Knee Surg Relat Res 2015; 27: 228-232, DOI: 10.5792/ksrr. 2015.27.4.228.

12. Hawker GA, Mian S, Kendzerska T, French M. Measures of adult pain: Visual Analog Scale for Pain (VAS Pain), Numeric Rating Scale for Pain (NRS Pain), McGill Pain Questionnaire (MPQ), Short-Form McGill Pain Questionnaire (SF-MPQ), Chronic Pain Grade Scale (CPGS), Short Form-36 Bodily Pain Scale (SF-36 BPS), and Measure of Intermittent and Constant Osteoarthritis Pain (ICOAP). Arthritis Care Res (Hoboken) 2011; 63 Suppl 11: S240-S252, DOI:10.1002/acr.20543.

13 Altman RD, Bedi A, Karlsson J, et al. Product differences in intraarticular hyaluronic acids for osteoarthritis of the knee. Am J Sports Med 2016; 44: 2158-2165, DOI: 10.1177/0363546515609599.

14. Migliore A, Tormenta S, Martin LS, et al. Open pilot study of ultrasound-guided intra-articular injection of hylan G-F 20 (Synvisc) in the treatment of symptomatic hip osteoarthritis. Clin Rheumatol 2005; 24: 285-289, DOI: 10.1007/s10067-004- 1009-1.

15. Steidle-Kloc E, Dannhauer T, Wirth W, Eckstein F. Responsiveness of infrapatellar fat pad volume change to body weight loss or gain: data from the osteoarthritis initiative. Cells Tissues Organs 2018; 205: 53-62, DOI: 10.1159/000485833.

16. Nishimuta JF, Levenston ME. Meniscus is more susceptible than cartilage to catabolic and anti-anabolic effects of adipokines. Osteoarthritis Cartilage 2015; 23: 1551-1562, DOI: 10.1016/ j.joca.2015.04.014.

17. Dargel J, Feiser J, Gotter M, et al. Side differences in the anatomy of human knee joints. Knee Surg Sports Traumatol Arthrosc 2009; 17: 1368-1376, DOI: 10.1007/s00167-009-0870-5.

18. Boesen M, Jensen KE, Torp-Pedersen S, et al. Intra-articular distribution pattern after ultrasound-guided injections in wrist joints of patients with rheumatoid arthritis. Eur J Radiol 2009; 69: 331-338, DOI: 10.1016/j.ejrad.2007.08.037.

19. Honvo G, Reginster J, Rannou F, et al. Safety of intra-articular hyaluronic acid injections in osteoarthritis: outcomes of a systematic review and meta-analysis. Drugs Aging 2019; 36 (Suppl 1): 101-127, DOI: 10.1007/s40266-019-00657-w. 